\title{
El caribe colombiano: entre la construcción de paz y la persistencia del conflicto ${ }^{1}$
}

\section{Colombian Caribbean Region: between peacebuilding and the persistence of conflict}

DOI: https://doi.org/10.17981/juridcuc.15.1.2019.01

Fecha de Recepción: 02/04/2019 Fecha de Aceptación: 16/04/2019

\author{
Luis Trejos Rosero, iD \\ Universidad del Norte (Colombia) \\ trejosl@uninorte.edu.co \\ Reynell Badillo Sarmiento \\ Universidad del Norte (Colombia) \\ reynellb@uninorte.edu.co \\ Yiseth Irreño Quijano iD \\ Universidad del Norte (Colombia) \\ yirreno@uninorte.edu.co
}

Para citar este artículo:

Trejos, L., Badillo, R. e Irreño, Y. (2019). El caribe colombiano: entre la construcción de paz y la persistencia del conflicto. JURÍDICASCUC, 15(1).9-46. http://dx.doi.org/10.17981/juridcuc.15.1.2019.01

\section{Resumen}

El Caribe colombiano alberga 4 de las 16 subregiones priorizadas para la realización de Programas de Desarrollo con Enfoque Territorial (PDET). Los PDET surgieron del Acuerdo de Paz firmado entre el Gobierno colombiano y la exguerrilla de las FARC a fin de transformar las condiciones de los territorios víctimas del conflicto armado. Sin embargo, algunas de esas subregiones aún presentan expresiones de violencia armada que podrían impedir la consecución de la paz territorial y, por el contrario, podrían contribuir a perpetuar esas condiciones de conflictividad armada. El presente trabajo pretende analizar estas cuatro subregiones desde un estudio comparado a través de tres variables de análisis: presencia de rentas ilegales, presencia de actores armados y grado de afectación derivado del conflicto armado, todas durante el periodo enero-septiembre del 2018. Se encuentra que, aunque las subregiones como Sierra Nevada-Perijá y Montes de María no presentan situaciones de alta conflictividad, hay retos de carácter consensual que pueden retrasar e, incluso, sabotear la implementación de los PDET. Por su parte, las subregiones Sur de Córdoba y Sur del Bolívar, por su extensión, tienen menos retos consensuales, pero un panorama de violencia que haría aún más complejo transitar hacia el posconflicto debido a la presencia de actores que buscarán sabotear el proceso (Clan del Golfo, Los Caparrapos y Disidencias de las FARC). Palabras clave: Programas de Desarrollo con Enfoque Territorial; conflicto armado; construcción de paz; Región Caribe.

\section{Abstract}

Four of the 16 sub-regions prioritized for the implementation of Territorial Approach Development Programs (PDET) are located in the Colombian Caribbean region. PDET arose from the Peace Agreement signed between the Colombian Government and the former armed group FARC in order to transform the conditions of the territories that were victims of the armed conflict. However, some of these sub-regions still show expressions of armed violence that could impede the achievement of territorial peace and, on the contrary, could contribute to perpetuate these conditions of war. This paper attempts to analyze these four subregions from a comparative study through three analysis variables: presence of illegal rents, presence of armed actors and degree of affectation derived from the armed conflict, all during the January-September period of 2018. The authors found that although subregions such as Sierra Nevada-Perijá and Montes de María do not face important problems of violence, there are challenges of consensual nature that may delay and even sabotage the implementation of PDET. For their part, the southern subregions of Córdoba and southern Bolívar have fewer consensual challenges, but a panorama of violence that would make it even more complex to move towards post-conflict due to the presence of actors who will seek to sabotage the process (Clan del Golfo, Los Caparrapos and Disidents from FARC armed group).

Keywords: Territorial Approach Development Programs; armed conflict; peacebuilding; Colombian Caribbean Region.

\footnotetext{
${ }^{1}$ Artículo derivado del proyecto "Ciudadanía Rural» financiado por el Centro de Pensamiento UNCaribe.
} 


\section{Introducción}

La firma de los Acuerdos de Paz entre el Gobierno colombiano y la otrora guerrilla de las Fuerzas Armadas Revolucionarias de Colombia (FARC) abrió el camino para que se diera una ampliación de la oferta institucional en zonas históricamente afectadas por la violencia armada en el Caribe colombiano. No obstante, la paz territorial que este acuerdo pretendía construir ha tenido que enfrentar retos de gran envergadura como el asesinato sistemático de líderes sociales, el fortalecimiento de organizaciones armadas como el Ejército de Liberación Nacional (ELN) y el Clan del Golfo, y la aparición de nuevos grupos violentos como Los Caparrapos en el sur de Córdoba, probablemente financiados por carteles mexicanos. Asimismo, en dos de las cuatro subregiones priorizadas para la puesta en marcha de los Programas de Desarrollo con Enfoque Territorial (PDET) hay presencia activa de actores armados $^{2}$ además de infracciones al Derecho Internacional Humanitario (DIH) y rentas ilegales.

De esta forma, el Caribe colombiano se encuentra frente a dos escenarios antagónicos: por un lado, la región tiene ante sí la necesidad de poner en marcha la construcción de la paz e implementar lo pactado en el "Acuerdo del Teatro Colón", mientras por otro, se enfrenta a la persistencia de la violencia armada que, en lugar de disiparse, se ha transformado ${ }^{3}$. En consecuencia, el presente trabajo pretende analizar las cuatro subregiones priorizadas en el Caribe colombiano para el desarrollo de los PDET a través de sus condiciones de violencia en aras de evidenciar los retos que enfrentará la puesta en marcha de estos proyectos.

\footnotetext{
${ }^{2}$ Estas regiones son los Montes de María, la Sierra Nevada y el Perijá; el Sur de Bolívar y el Sur de Córdoba (Estas dos últimas son las que presentan serios problemas de orden público).

${ }^{3}$ Lo que se ha venido presentando en la actualidad en esta región de Colombia, es una mutación de los tipos "tradicionales" de violencia, al pasar de una violencia contraestatal paraestatal a una muy difusa violencia de tipo criminal, ligada al narcotráfico y la extorsión con un alto poder corruptor y dirigida específicamente contra la sociedad civil (Trejos, 2017).
} 
Para lo anterior, en este artículo se comenzará por describir qué son los PDET, cuáles son sus objetivos, los criterios utilizados para priorizar municipios y cuáles municipios serán intervenidos por estos programas. Acto seguido, se esbozará el análisis de cada una de las subregiones priorizadas a partir de tres variables: la presencia de rentas ilegales (cultivos ilícitos y/o minería ilegal), la presencia de actores armados ilegales (Grupos Armados Organizados - GAO o guerrillas - Ejército de Liberación Nacional, ELN) y el grado de afectación derivado del conflicto armado (acciones armadas presentadas durante el 2018 y las infracciones al Derecho Internacional Humanitario (DIH) ocurridas durante el mismo año ${ }^{4}$. Finalmente, se analizarán las subregiones a partir de la presencia y variedad de Consejos Comunitarios y Resguardos Indígenas en aras de plantear retos de tipo consensual que se puedan presentar a la hora de planificar el territorio a largo plazo.

En ese sentido, se trata de una investigación comparativa, como lo establecen Marsh y Stoker (1997), al analizar las condiciones de un número limitado de entidades (4 subregiones para este caso) a fin de establecer inferencias políticas y teóricas a través de su contraste. Asimismo, tal como lo mencionan Esser y Vliegenthart (2017), se han seleccionado "unidades de nivel micro" representadas por "unidades subnacionales" (p. 2). Estas cuatro subregiones van a ser contrastadas a la luz de las variables explicadas anteriormente. Y como recomienda Landman (2000), esas variables se pueden aplicar a otros casos de estudio a fin de elaborar un análisis comparado.

Los investigadores consideran que las subregiones del sur de Córdoba y el sur de Bolívar enfrentarán mayores problemas a la hora de implementar los PDET debido a las difíciles

\footnotetext{
${ }^{4}$ Estas variables fueron observadas a la luz de la base de datos del Observatorio de Dinámicas del Conflicto Armado en el Caribe Colombiano del Centro de Pensamiento UNCaribe de la Universidad del Norte durante el periodo enero-septiembre del 2018.
} 
condiciones de violencia armada que enfrentan. En estas subregiones, la amplia presencia de actores armados, el asedio a los liderazgos sociales y el crecimiento de las rentas ilegales representan serios retos para lograr que los acuerdos alcanzados se materialicen y alcancen sus principales objetivos. No obstante, no parece que las condiciones de violencia afecten necesariamente el proceso de concertación de los PDET. Por el contrario, la heterogeneidad de las subregiones de Montes de María y Sierra Nevada - Perijá podrían plantear mayores retos debido al tamaño de las subregiones priorizadas y a las condiciones heterogéneas entre territorios y comunidades.

\section{Programas de Desarrollo con Enfoque Territorial: Aproximación descriptiva}

El Punto 1 de los Acuerdos de Paz pactados entre el Gobierno colombiano y la exguerrilla de las FARC contiene la Reforma Rural Integral (RRI). Esta reforma reconoce los diferentes problemas sociales, económicos, étnicos y de desarrollo que presenta el campo colombiano y se propone hacer una transformación estructural de este. Busca promover la participación, llevar desarrollo socioeconómico, proveer bienes y servicios públicos como agua, electricidad, salud y educación en los territorios, dar igualdad de oportunidades a hombres y mujeres, garantizar la seguridad alimentaria y reconocer los derechos de las diferentes comunidades étnicas que habitan el territorio (Acuerdos Final, 2016).

Para lograr esos fines, la RRI planteó la priorización de los territorios que más sufrieron el conflicto armado colombiano teniendo en cuenta variables como afectaciones causadas por las acciones armadas, el índice de pobreza multidimensional, el bajo desempeño integral de sus administraciones y la presencia de rentas ilícitas; con el fin de ejecutar una serie de proyectos de impacto local y regional por medio de unos Programas de Desarrollo con Enfoque Territorial (PDET) que deberán: 
... tener un enfoque territorial, diferencial y de género que implica reconocer y tener en cuenta las necesidades, características y particularidades económicas, culturales y sociales de los territorios, de las mujeres en todo su ciclo vital, de las comunidades rurales y de grupos en condiciones de vulnerabilidad, garantizando la sostenibilidad socio-ambiental. (Acuerdo de paz, 2017, p. 11).

Estos PDET, según la Oficina del Alto Comisionado para la $\mathrm{Paz}$ (s.f.), son el instrumento de planificación que orienta las acciones de las administraciones departamentales, distritales y municipales durante un período de gobierno que está contemplado en el Decreto 893 de 2017. Por medio de ese decreto se definen las zonas priorizadas con PDET, se establecen los Planes de Acción para la Trasformación Regional (PATR) en que se fundan los PDET, se reglamenta la participación de la ciudadanía en la formulación de los planes y programas y se vinculan los PDET con el Plan Nacional de Desarrollo.

La definición de las zonas priorizadas se hizo a partir de los siguientes criterios:

- Los niveles de pobreza, en particular de pobreza extrema y necesidades básicas insatisfechas.

- El grado de afectación derivado del conflicto.

- La debilidad de la institucionalidad administrativa y de la capacidad de gestión, la presencia de cultivos de uso ilícito y de otras economías ilegítimas (Decreto 893, 2017, p. 9).

Estos criterios fueron establecidos según el Punto 1.2 del Acuerdo de Paz, y fueron reafirmados por el decreto mencionado anteriormente. De esta forma se delimitaron 170 municipios a intervenir, agrupados en 16 subregiones. Cada subregión con un PDET; es decir, se formularán 16 en Colombia. De esas 16 subregiones 4 pertenecen al Caribe colombiano, que son: los Montes de María, la Sierra Nevada y Perijá, el Sur de Bolívar y el Sur de Córdoba. Para liderar la creación de estos PDET se crea la Agencia para la Renovación del Territorio (ART) encargada de coordinar la intervención del Gobierno Nacional en los territorios (García, Irreño y Gutiérrez, 2018). 
Tabla 1

Las 16 subregiones priorizadas

\begin{tabular}{c}
\hline Subregión \\
Alto Patía-Norte del Cauca \\
Aajo Cauca y Nordeste Antioqueño \\
Catatumbo \\
Choco \\
Cuenca del Caguán y piedemonte caqueteño \\
Macarena-Guaviare \\
Sur del Tolima \\
Pacífico Medio \\
Pacífico y Frontera Nariñese \\
Putumayo \\
Urabá antioqueño \\
Sur de Bolívar \\
Sur de Córdoba \\
Montes de María \\
Sierra Nevada-Perijá
\end{tabular}

Fuente: Elaboración propia a partir del Decreto 893 (2017).

La metodología planteada por la ART para el diseño de los PDET en los 170 municipios consistió en un trabajo que se dio, y se sigue dando, en tres momentos: el primero es el veredal, luego el municipal y por último el regional. En el nivel veredal, se realizaron un conjunto de preasambleas veredales, dividiendo el territorio rural de los municipios en núcleos veredales cuyo número de participantes y núcleos dependerá de las características de los territorios. En cada núcleo veredal se trabajó con la comunidad alrededor de ocho pilares: 1) Ordenamiento social de 
la propiedad rural y uso del suelo. 2) Reactivación económica y producción agropecuaria. 3) Educación rural. 4) Vivienda, agua potable y saneamiento. 5) Salud rural. 6) Derecho a la alimentación. 7) Reconciliación, convivencia y paz. 8) Infraestructura y adecuación de tierras. Estos pilares se distribuyeron por mesas, es decir, en las preasambleas había ocho mesas y los asistentes se sentaban en la mesa que más le interesaba.

La dinámica consistia en dar en cada pilar a los miembros de las comunidades, orientados bajo las directrices de un facilitador $^{5}$, la facilidad de nombrar y enumerar cuales eran las oportunidades y problemáticas de su territorio y plantear iniciativas que dieran solución a dichas problemáticas. Luego, esos resultados se expusieron en unas carteleras para ser socializados con todos los asistentes. Para finalizar, las personas que trabajaron en cada pilar seleccionaron a un representante, con el fin de que asistiera al momento municipal. De estas preasambleas, se derivaron pactos veredales (un conjunto de propuestas para los ocho pilares).

El Pacto Veredal fue llevado al momento municipal para ser deliberado por todos los representantes escogidos en los núcleos veredales y priorizar las iniciativas planteadas en un período de tiempo de corto, mediano y largo plazo. Por último, en el momento regional se formularán los Planes de Acción para la Transformación Regional (PATR).

Tabla 2

Plazos de las Iniciativas

\begin{tabular}{cc}
\hline Plazo & Años \\
\hline Corto & De 1 a 2 años \\
Mediano & De 2 a 5 años \\
Largo & De 5 a 10 años \\
\hline
\end{tabular}

Fuente: Elaboración propia.

\footnotetext{
${ }^{5}$ La figura del facilitador consistia en la capacidad de un miembro de la ART para encargarse de orientar la discusión que se daba en cada uno de los pilares.
} 
Tabla 3

Momentos de la construcción de los PDET

\begin{tabular}{|c|c|c|}
\hline Fase/nivel & Eventos de la fase/nivel & $\begin{array}{l}\text { Resultados de la } \\
\text { fase/nivel }\end{array}$ \\
\hline Veredal & $\begin{array}{ll}\text { - } & \text { Alistamiento } \\
\text { - } & \text { Preasamblea } \\
\text { - } & \text { Grupo Motor } \\
\text { - } & \text { Asamblea }\end{array}$ & $\begin{array}{c}\text { Pacto Comunitario } \\
\text { para la } \\
\text { Transformación } \\
\text { Regional (PCTR) }\end{array}$ \\
\hline Municipal & $\begin{array}{ll}\text { - } & \text { Encuentro de Diálogos } \\
& \text { Preparatorios } \\
\text { - } & \text { Precomisión Municipal } \\
\text { - } & \text { Comisión Municipal }\end{array}$ & $\begin{array}{l}\text { Pacto Municipal para } \\
\text { la Transformación } \\
\text { Regional (PMTR) }\end{array}$ \\
\hline Regional & $\begin{array}{ll}\text { - } & \text { Encuentro de Diálogos } \\
\text { - } & \text { Preparatorios } \\
\text { Precomisiones (Mesas de } \\
\text { trabajo por Pilar) } \\
\text { - } \quad \text { Comisión Subregional } \\
\text { Encuentro de Secretaría } \\
\text { Técnica para la redacción y } \\
\text { formalización de PATR }\end{array}$ & $\begin{array}{l}\text { Plan de Acción para } \\
\text { la Transformación } \\
\text { Regional (PATR) }\end{array}$ \\
\hline
\end{tabular}

Fuente: Misión de Observación Electoral-MOE (2018).

En esos tres momentos de construcción de los PDET se busca fomentar la participación ciudadana y los liderazgos sociales, al ser estos los que expusieron las problemáticas de sus municipios y plantearon iniciativas para solucionarlas dentro de las mismas comunidades. No obstante, los proyectos que surjan de la comunidad serán luego revisados por personal técnico que evaluará su viabilidad en la fase subregional, tal como se puede observar en la Tabla 3. Bajo esta lógica, los PDET se consideran un instrumento de planeación participativa, por ser el que "combina, por tanto, ingredientes técnicos y sociopolíticos" (Velásquez y González, 2003, p. 25) y esta es una característica propia de la planeación participativa.

Sin embargo, para que los PDET puedan aportar a la consecución de los objetivos planteados por la RRI, antes deben superar una serie de obstáculos en los municipios a intervenir. 
Dado que en algunos de ellos persisten dinámicas de violencia, grupos al margen de la ley y rentas ilegales, se hace necesario considerar estas variables e intentar comprender las posibles formas en que podrian afectar el proceso de construcción de paz territorial.

\section{Caribe colombiano:}

Nuevas y viejas formas de violencia que amenazan la construcción de la paz territorial

El Caribe colombiano se conforma administrativamente por 7 departamentos continentales (La Guajira, Magdalena, Cesar, Bolívar, Atlántico, Córdoba y Sucre) y 1 departamento insular (San Andrés y Providencia). La extensión territorial de esta región corresponde al 11,6\% de la superficie total del país y, según el Observatorio del Caribe colombiano (2013), puede ser dividida en cinco subregiones similares en términos climáticos y/o geográficos: la península de La Guajira, la Sierra Nevada de Santa Marta, la depresión del Bajo Magdalena, la depresión Momposina y la planicie del Caribe.

Otros autores como Daniels (2012) prefieren una tipología de la región que, trascendiendo las homogeneidades geográficas, se centre en las dinámicas socioeconómicas. Así, este considera que existen tres subregiones: la costera, con un importante dinamismo económico; el área interior, que no ha podido desarrollar completamente su estructura productivoeconómica; y las subregiones afectadas directamente por el conflicto, entre las que se encuentran los Montes de María, la Sierra Nevada de Santa Marta, y el sur de los departamentos de Bolívar y Córdoba. Como puede ser observado, la categorización de Daniels (2012), se acerca a la división subregional que esbozó el Decreto 893. A continuación, se hace un breve repaso por el contexto de violencia en cada subregión priorizada, y se analizan las condiciones actuales de violencia, y consecuentemente, los retos que cada una de ellas deberá afrontar para su materialización. 
Montes de María:

De teatro de operaciones de actores ilegales a ejemplo de reconciliación

La subregión de los Montes de María se extiende territorialmente por $6.466 \mathrm{~km}^{2}$ del Caribe y comprende a 15 municipios de dos departamentos de Colombia, Bolívar ${ }^{6}$ ( $58,9 \%$ del territorio) y $\operatorname{Sucre}^{7}(42,1 \%$ del mismo). La región es atravesada por tres ejes: la carretera Troncal de Occidente que comunica los municipios de San Juan Nepomuceno, San Jacinto, Carmen de Bolívar, Ovejas y Los Palmitos, y la Troncal del Caribe que comunica los municipios de Marialabaja, San Onofre y Toluviejo, permitiendo la comunicación de los Montes de María con el norte y el sur del país, y la Transversal de los Contenedores que une los municipios de Plato (Magdalena), Zambrano, El Carmen de Bolívar y que permite la comunicación de los Montes de María con el oriente y el centro del país, a través de la Troncal de Oriente. En el centro de estos ejes, se encuentra ubicado el municipio de El Carmen de Bolívar (Organización de las Naciones Unidas-ONU. Programa de las Naciones Unidas para el Desarrollo-PNUD, 2011).

En esta subregión, el conflicto armado irrumpe durante la década de los 80, luego de la VII Conferencia de las FARC en 1982, donde se le da el nombre de "Plan Estratégico" o "Campaña Bolivariana para una nueva Colombia" (Aguilera, 2013). La entonces guerrilla decide extender sus zonas de influencia y desdoblar varios de sus frentes. De esta forma, se crearon los Frentes 35 "Antonio José de Sucre" y 37 "Benkos Biohó", el primero con radio de acción en Sucre y el segundo en Bolívar (Trejos, 2016).

Un año después, en la "Reunión Nacional de héroes y mártires de Anori" del ELN, este grupo armado decide crear el

\footnotetext{
${ }^{6}$ Municipios de Bolívar: Córdoba, El Carmen de Bolívar, El Guamo, María la Baja, San Jacinto, San Juan Nepomuceno y Zambrano.

${ }^{7}$ Municipios de Sucre: Chalán, Colosó, Los Palmitos, Morróa, Ovejas, San Antonio de Palmito, San Onofre, Sincelejo y Toluviejo.
} 
Frente José Solano Sepúlveda, que tenía al Frente Jaime Bateman Cayón actuando en los Montes de María. Además, la compañía "Ernesto Che Guevara" del extinto Ejército Revolucionario del Pueblo (ERP) también se asentó en la serranía de San Jacinto, llegando a operar en ambos departamentos (Daniels, Menco, Jiménez y Peralta, 2011, Trejos, 2018a).

Con respecto al paramilitarismo, si bien las Autodefensas Unidas de Colombia (AUC) llegaron a la región aproximadamente en 1997, desde finales de los años 70 y comienzos de los 80 pequeños grupos de hombres fueron armados por dueños de fincas que sentían amenazadas sus propiedades debido a la búsqueda de titulación de predios por parte de la Asociación de Usuarios Campesinos (ANUC). Así, estos grupos terminaron amedrentando dirigentes sociales bajo la excusa de una supuesta lucha contrainsurgente (Verdad Abierta, 2010):

En la zona urbana de El Carmen de Bolívar estaba La Mano Negra. En Sincelejo, había una banda llamada La Cascona, que incluso figuraba en la lista de paramilitares que reveló en octubre de 1987 el entonces ministro de Gobierno César Gaviria ante el Congreso. En esa misma lista, aparecía la banda 'Muerte a Secuestradores y Comunistas' en el departamento de Bolívar. Y estaban Los RR en San Juan Nepomuceno, Los Benítez en San Pedro y los Encapuchados de Colosó.

Sin embargo, fue en la segunda mitad de los años 90 cuando el conflicto armado se escaló en las tierras montemarianas dejando un saldo de 56 masacres, más de 200.000 desplazados, más de 2.000 homicidios y cerca de 50.000 hectáreas de tierra abandonadas (Verdad Abierta, 2010, Daniels et al., 2011).

A mediados de la década del 2000 se desmovilizaron los Frentes Canal del Dique, Golfo de Morrosquilo y Sabanas de Sucre y Córdoba del Bloque Héroes Montes de María de las AUC, reportando 594 combatientes. Con esto, permanecieron en el territorio las guerrillas de las FARC, el ERP y el ELN y nuevos grupos armados residuos de las AUC; sin embargo, los frentes 35 y 37 de las FARC pasaron a producir cerca del $80 \%$ de la actividad violenta (Observatorio de Derechos Humanos y 
Tabla 4

Conflictividad en la subregión Montes de María en el 2018

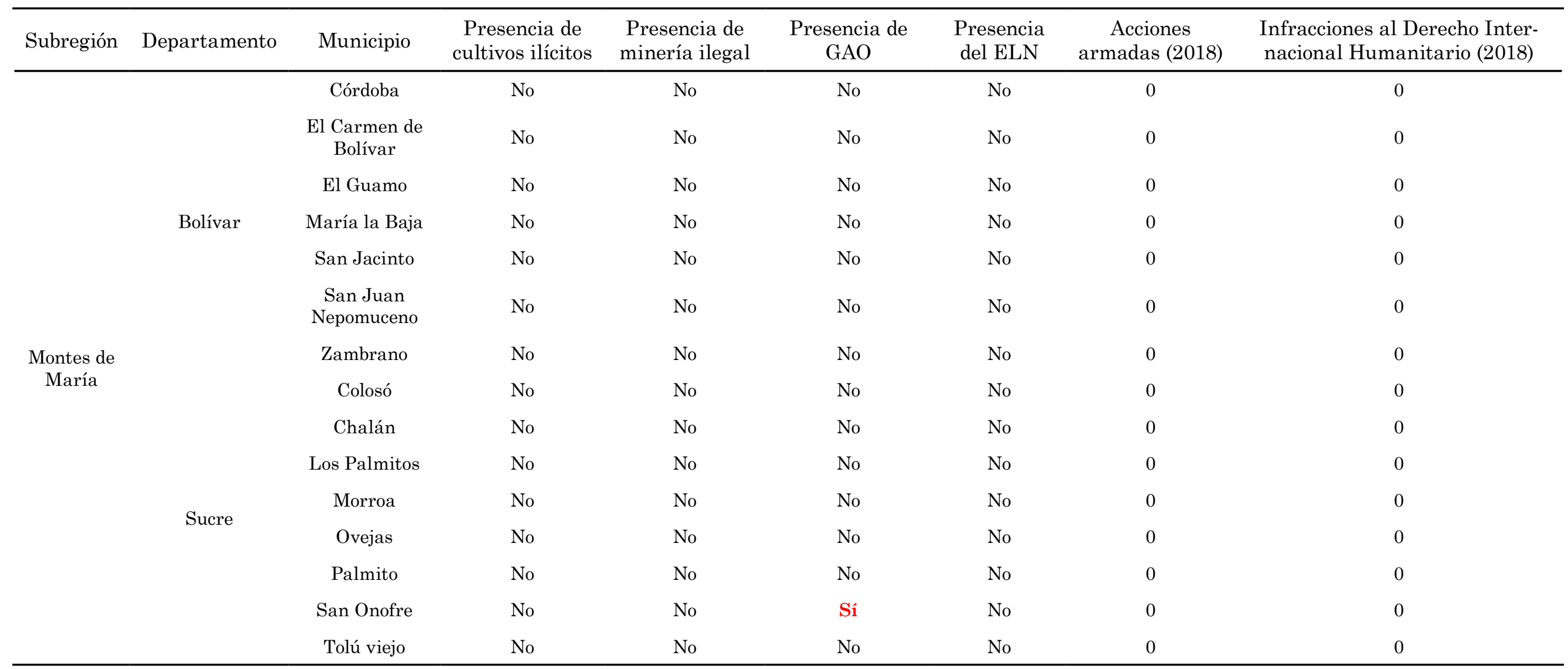

Fuente: Elaboración propia a partir del Centro de Pensamiento UNCaribe (2018).

Derecho Internacional Humanitario, 2008). Ya para el año 2010, se consideraba que la reducción de las muertes daba cuenta de una relativa calma en medio del conflicto, y se registra un amplio número de organizaciones de la sociedad civil que trabajaban (y aún trabajan) por construir una cultura de paz (PNUD, 2010).

En la Tabla 4 es posible notar en términos generales como en la región no se desarrollan acciones armadas desde hace varios años y tampoco se han presentado infracciones al Derecho Internacional Humanitario durante el 2018, no existe presencia de cultivos ilícitos ni minería ilegal, el ELN no está presente en la subregión, y con excepción de San Onofre (Sucre), no hay presencia de grupos armados organizados.

En la Alerta Temprana Nº61-18 (El Heraldo.com, 2018) se considera a los cerca de 5.900 pobladores de San Onofre en alto riesgo debido a la presencia de las autodenominadas Autodefensas Gaitanistas de Colombia (AGC), conocidas como Clan del Golfo. Además, el documento cuestiona la inacción de las autoridades locales para contener esta amenaza, y por el contrario, asegura la comunidad una percepción de tolerancia con el accionar del grupo criminal. 
Si bien esta situación por el momento afecta únicamente a San Onofre, no debería considerarse que la subregión de los Montes de María ha superado totalmente el conflicto armado en todas sus dimensiones. La mencionada amenaza del Clan del Golfo podría tomarse como una muestra de que las condiciones de inequidad social y pobreza en territorios estratégicos ${ }^{8}$ como estos, pueden contribuir a la permanencia del conflicto. No obstante, al menos en el presente, los Montes de María parece tener condiciones adecuadas para la puesta en marcha de los PDET en términos de situaciones provenientes del conflicto armado.

\section{Sierra Nevada - Perijá: ¿Libre de conflicto?}

La subregión de la Sierra Nevada - Perijá está conformada por quince (15) municipios, al igual que la subregión montemariana; y sin embargo, esta presenta una heterogeneidad más marcada al comprender municipios de tres departamentos: Cesar, La Guajira y Magdalena. Asimismo, como puede ser deducido de su nombre, difícilmente puede considerarse una subregión unificada y resultaría mucho más acertado comprender la presencia de dos espacios diferenciados: el de la Sierra Nevada y el de los municipios con territorios en la serranía del Perijá.

Los municipios La Paz, Manaure Balcón del Cesar, San Diego, Agustín Codazzi, La Jagua de Ibirico, Becerril (departamento de Cesar), Fonseca, San Juan del Cesar (departamento de La Guajira) se encuentran ubicados geográficamente en la Serranía del Perijá. Por su parte, Valledupar, Pueblo Nuevo (al norte del Cesar), Dibulla (al sur de La Guajira), Santa Marta, Aracataca, Ciénaga y Fundación (departamento de Magdalena) se encuentran dentro de La Sierra Nevada de Santa Marta (Aguilera, 2016).

En la Sierra Nevada de Santa Marta hicieron presencia las guerrillas de la FARC-EP a través de los frentes 19 (José Pru-

\footnotetext{
${ }^{8}$ Montes de María esta conformada por los municipios de San Juan Nepomuceno, San Jacinto, Carmen de Bolívar, Ovejas y Los Palmitos (Troncal de Occidente), los municipios de Marialabaja, San Onofre y Toluviejo (Troncal del Caribe) y los municipios de Plato (Magdalena), Zambrano, El Carmen de Bolívar (Troncal de Oriente) (PNUD, 2011).
} 
dencio Padilla) y 59 (Resistencia Wayúu), y el ELN a través de los frentes Gustavo Palmezano Ojeda, 6 de Diciembre, Francisco Javier Castaño, y Domingo Barrios, y de las compañías Augusto Montes y Héroes de las Bananeras; por su parte, en la Serranía del Perijá hizo presencia el Frente 41 (Cacique Upar) de las FARC-EP, los frentes José Manuel Martínez Quiroz (concentrado en el departamento del Cesar) y Luciano Ariza (actuando primordialmente en La Guajira) y la compañía Óscar Enrique Sánchez Caicedo del ELN (Trejos, 2018a).

Por su parte, el paramilitarismo actuó en la Sierra Nevada de Magdalena a través del Frente Resistencia Tayrona, adscrito al Bloque Norte de las AUC, que dejó cerca de 67 mil víctimas en la región; no obstante, desde los años 80 se conforman grupos paramilitares como el liderado por Hernán Giraldo, quien, ante la imposibilidad de combatir efectivamente la llegada del Bloque Norte termina doblegándose y actuando como jefe del frente mencionado (Verdad Abierta, 2014). En la Serranía del Perijá actuó principalmente el frente Mártires del Valle de Upar, adscrito también al Bloque Norte de las AUC, aunque también se registró presencia del frente Juan Andrés Álvarez (en Agustín Codazzi y la Jagua de Ibirico), y más hacia el sur del Cesar el Frente Resistencia Motilona (Arias, 2010).

En cuanto al contexto contemporáneo de conflicto, si bien, la situación en términos generales no evidencia altos niveles de violencia armada, sí existe una mayor conflictividad en comparación con la subregión de Montes de María. En cuanto a la primera categoría (presencia de rentas ilegales), el único municipio que aparece afectado es Santa Marta, capital del departamento del Magdalena. No obstante, en el informe del 2018 la Oficina de Naciones Unidas contra la Droga y el Delito (UNODC por sus siglas en inglés) se puede observar que la tendencia en este departamento es a la baja, ya que mientras en el 2016 se presentaban 35 hectáreas sembradas de coca, en el 2017 la cifra llegó a 8 hectáreas, representando apenas el 0,005\% del total en el país (UNODC, 2018). 
Tabla 5

Conflictividad en la subregión Sierra Nevada-Perijá en el 2018

\begin{tabular}{|c|c|c|c|c|c|c|c|c|}
\hline Subregión & Departamento & Municipio & $\begin{array}{l}\text { Presencia de } \\
\text { cultivos ilícitos }\end{array}$ & $\begin{array}{l}\text { Presencia de } \\
\text { minería ilegal }\end{array}$ & Presencia de GAO & $\begin{array}{l}\text { Presencia } \\
\text { del ELN }\end{array}$ & $\begin{array}{c}\text { Acciones } \\
\text { armadas (2018) }\end{array}$ & $\begin{array}{c}\text { Infracciones al } \\
\text { Derecho Internacional } \\
\text { Humanitario (2018) }\end{array}$ \\
\hline & & Valledupar & No & No & No & No & 0 & 0 \\
\hline & & $\begin{array}{l}\text { Agustín } \\
\text { Codazzi }\end{array}$ & No & No & No & No & 0 & 0 \\
\hline & & Becerril & No & No & No & No & 0 & 0 \\
\hline & $C_{0}$ & $\begin{array}{l}\text { La Jagua de } \\
\text { Ibirico }\end{array}$ & No & No & No & No & 0 & 0 \\
\hline & Cesdal & Pueblo Bello & No & No & No & No & 1 & 1 \\
\hline & & $\mathrm{LaPaz}$ & No & No & No & No & 1 & 0 \\
\hline & & San Diego & No & No & No & No & 0 & 0 \\
\hline \multirow[t]{8}{*}{$\begin{array}{r}\text { Sierra } \\
\text { Nevada - } \\
\text { Perijá }\end{array}$} & & $\begin{array}{l}\text { Manaure } \\
\text { Balcón del } \\
\text { Cesar }\end{array}$ & No & No & No & No & 1 & 0 \\
\hline & & Dibulla & No & No & No & Sí & 0 & 0 \\
\hline & La Guajira & Fonseca & No & No & No & No & 0 & 0 \\
\hline & & $\begin{array}{l}\text { San Juan del } \\
\text { Cesar }\end{array}$ & No & No & No & No & 0 & 0 \\
\hline & & Santa Marta & Sí & No & No & Sí & 0 & 0 \\
\hline & Magdalena & Aracataca & No & No & No & No & 0 & 1 \\
\hline & & Ciénaga & No & No & No & No & 0 & 0 \\
\hline & & Fundación & No & No & No & No & 0 & 0 \\
\hline
\end{tabular}

Fuente: Elaboración propia a partir del Centro de Pensamiento UNCaribe (2018).

Sobre la presencia de actores armados, es necesario mencionar que en ninguno de los municipios hacen presencia GAO, y solamente en Santa Marta (específicamente en zona montañosa de la Sierra Nevada) y Dibulla (La Guajira) se ha avistado presencia del ELN.
Lo anterior se verifica por medio de la Alerta Temprana $\mathrm{N}^{\circ} 045$ 18 (Defensoría del Pueblo, 2018b), en la que el ente advirtió sobre la amenaza a la que están sometidos pueblos indígenas como los Kogui, Malayo, Arhuaco y Kankuamos debido a la reaparición de 
la guerrilla pero, sobre todo, a la existencia de Los Pachencas, un Grupo Delictivo Organizado (GDO) que surgió en el 2013 al mando de quien, un año antes, había sido capturado por ser el cabecilla del Clan del Golfo en la Sierra Nevada de Santa Marta (Iguaran, 2017). Asimismo, según la Fundación Paz y Reconciliación citada por Ávila (2018), alrededor del $60 \%$ de la actividad comercial en zona rural de Santa Marta y el $30 \%$ en su casco urbano está siendo extorsionada por este grupo delictivo. En la misma Alerta Temprana también aparece el municipio de Ciénaga pero no por la presencia del ELN sino de los Pachenca ${ }^{9}$. Además, muy cerca del corregimiento de Minca durante el año 2018, fue secuestrada una mujer, presuntamente por este GDO, siendo el segundo secuestro del año en la región Caribe; y en mayo, el Ejército reforzó su presencia allí debido a la posible amenaza del ELN (Urieles, 2018).

Por su parte, en lo referente al grado de afectación derivado del conflicto armado durante el año, se han presentado tres acciones armadas, todas por parte de la Fuerza Pública. En Manaure Balcón del Cesar fue capturado un cabecilla del Clan del Golfo, en La Paz y Pueblo Bello (Cesar) el Ejército descubrió y destruyó material de guerra perteneciente al frente Juan Manuel Martínez Quiroz del ELN.

Asimismo, se han presentado dos infracciones al DIH en esta subregión, tratándose en ambos casos de asesinatos de líderes sociales en Aracataca (Magdalena) y Pueblo Bello (Cesar). Este panorama muestra que, si se consideran por separado las subregiones de la Sierra Nevada y el Perijá, es posible observar que la primera tiene dinámicas de violencia más marcadas que la segunda. Sin embargo, no se trata de violencia a gran escala y, por el contrario, indicadores como el área sembrada de coca muestran que la tendencia no es al aumento en todas las variables.

\footnotetext{
${ }^{9}$ Estos datos no fueron incluidos en la Tabla 5 porque Los Pachenca no son considerados aún un GAO.
} 


\section{Sur de Bolívar: el conflicto persiste}

La tercera subregión por analizar comprende 6 municipios del departamento de Bolívar. Al tratarse de una subregión mucho más pequeña en términos de superficie, por lo tanto más homogénea, esta presenta mayores oportunidades para alcanzar acuerdos en la fase regional del PDET. No obstante, el panorama de violencia allí es mucho más complejo que en las anteriores subregiones.

En esta subregión, el ELN aún hace presencia a través del Frente de Guerra Darío de Jesús Ramírez Castro, al cual están adscritos los frentes Edgar Amílkar Grimaldos, Héroes y Mártires de Santa Rosa, José Solano Sepúlveda y Alfredo Gómez Quiñónes. Asimismo, en el Sur de Bolívar operan las Compañías Simón Bolívar y Mariscal Sucre. Por su parte, la exguerrilla de las FARC-EP hizo presencia a través de los frentes 24 y 37, adscritos a los bloques Magdalena Medio y Martín Caballero, respectivamente (Trejos, 2018b).

El paramilitarismo en la región actuó principalmente bajo la égida del Bloque Central Bolívar de las AUC, uno de los más grandes de la organización, a través de los frentes Santa Rosa del Sur y Nordeste Antioqueño, Bajo Cauca y Magdalena Medio (Observatorio de Paz y Conflicto-OPC, 2012; Gutiérrez, 2018).

Como puede ser evidenciado en la Tabla 6, el conflicto en el sur de Bolívar aún persiste. Con respecto a la presencia de rentas ilegales, todos los municipios a excepción de San Pablo tienen cultivos ilícitos, y en todos, menos en Cantagallo y San Pablo, se practica minería ilegal. Al contrastar esos datos con el reciente informe de la UNODC (2018) es posible observar que en el 2017 en este departamento se registraron 2.085 hectáreas sembradas de coca, más que en el año inmediatamente anterior, lo que representa un aumento del $51 \%$ y además, ha sido la cifra más alta desde el 2010 (en ese año había sembrados en el departamento 3.324 has de coca y en el 2017 aparecen 6.179 has). Así, Bolívar es el séptimo departamento con más hectáreas sembradas de coca y representa el $4 \%$ del total cultivado en el país, todo concentrado en el sur del departamento. 
Tabla 6

Conflictividad en la subregión Sur de Bolivar, 2018

\begin{tabular}{|c|c|c|c|c|c|c|c|c|}
\hline Subregión & Departamento & Municipio & $\begin{array}{l}\text { Presencia de } \\
\text { cultivos ilícitos }\end{array}$ & $\begin{array}{l}\text { Presencia de } \\
\text { minería ilegal }\end{array}$ & Presencia de GAO & Presencia del ELN & $\begin{array}{c}\text { Acciones } \\
\text { armadas } \\
(2018)\end{array}$ & $\begin{array}{c}\text { Infracciones al Derecho } \\
\text { Internacional Humanitario } \\
(2018)\end{array}$ \\
\hline \multirow{6}{*}{$\begin{array}{l}\text { Sur de } \\
\text { Bolívar }\end{array}$} & \multirow{6}{*}{ Bolívar } & Arenal & Sí & Sí & No & Sí & 1 & 0 \\
\hline & & Cantagallo & Sí & No & Sí & Sí & 2 & 1 \\
\hline & & Morales & Sí & Sí & No & Sí & 1 & 0 \\
\hline & & San Pablo & No & No & Sí & Sí & 1 & 1 \\
\hline & & $\begin{array}{c}\text { Santa Rosa } \\
\text { del Sur }\end{array}$ & Sí & Sí & Sí & Sí & 4 & 0 \\
\hline & & Simití & Sí & Sí & Sí & No & 1 & 0 \\
\hline
\end{tabular}

Fuente: Elaboración propia a través de información del Centro de Pensamiento UNCaribe (2018).

Con respecto a la presencia de actores armados, todos los municipios, a excepción de Simití, tienen presencia permanente del ELN, y solo Arenal y Morales no cuentan con presencia de GAO (en este caso, Clan del Golfo). Este panorama se explica mejor si se contrasta con las afectaciones derivadas del conflicto armado: en esta subregión se han presentado 10 acciones armadas hasta septiembre del 2018 (lo que representa cerca del $12 \%$ de todas las acciones armadas cometidas en la región Caribe), y 2 infracciones al DIH. El Gráfico $N^{\circ} 1$ permite observar las acciones armadas presentadas en las 4 subregiones proporcionalmente al total presentado hasta septiembre del 2018.

El ELN ha estado involucrado en todas las acciones armadas registradas, siendo 4 de ellas atentados, 5 acciones de la fuerza pública, y 1 enfrentamiento entre la guerrilla y el Ejército. Por su parte, las dos infracciones al DIH registradas fueron el asesinato de un líder social (Nixon Mutis en Cantagallo) y el de un exmiembro del ELN al recobrar la libertad.
- Acciones armadas en la subregión de Sierra Nevada y Perijá

- Acciones armadas en la subregión de Sur de Bolívar

Acciones armadas en la subregión de Sur de Cordoba

- Acciones armadas fuera de las subregiones

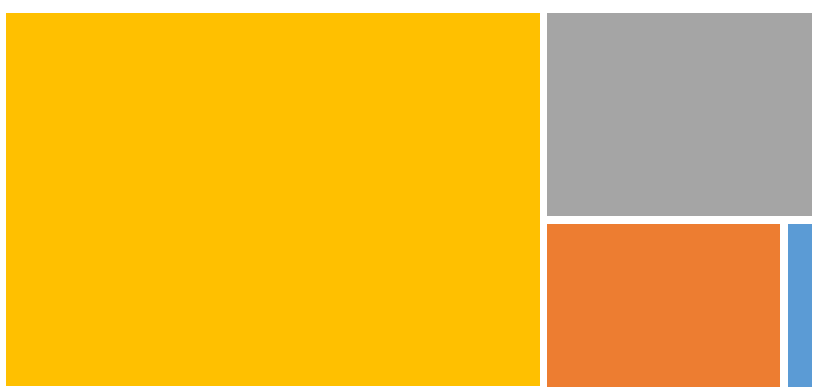

Figura 1. Acciones armadas por subregión: enero-septiembre, 2018. Fuente: Elaboración propia a partir del Centro de Pensamiento UNCaribe (2018). 


\section{Sur de Córdoba:}

La transformación de la violencia en manos del crimen organizado

Esta subregión es la más pequeña en términos de cantidad de municipios priorizados (solamente 5). Sin embargo, es asimismo, la que presenta mayores niveles de conflictividad. En esta subregión hizo presencia el Ejército Popular de Liberación (EPL) que para el año 1967 llegó a ubicarse en los límites entre los departamentos de Córdoba y Antioquia con el Frente Francisco Garnica y el comando urbano Pedro León Arboleda. Posteriormente, la guerrilla de las FARC se asentó en el sur de Córdoba a través de los frentes 18 y 58 , y en el futuro también harían presencia los frentes 5 y 36 (Irreño y Martínez, 2018).

Las AUC hicieron presencia en Córdoba con cuatro bloques: el Bloque Córdoba (dirigido por "Salvatore Mancuso"), el Bloque Héroes de Tolová (bajo el mando de "Don Berna"), el Bloque Mi- neros (dirigido por "Cuco Vanoy") y el Bloque Élmer Cárdenas (al mando de alias "El Alemán"). No obstante, mucho antes de la consolidación de las AUC, en Córdoba estaban presentes las Autodefensas Campesinas de Córdoba y Urabá (ACCU), y mucho antes ya se habían conformado otros grupos de autodefensas campesinas que terminaron sucumbiendo luego ante la llegada de las AUC (Observatorio de Derechos Humanos y Derecho Internacional Humanitario, 2009).

Con respecto a la violencia actual, esta subregión presenta el peor panorama entre las cuatro subregiones priorizadas para los PDET. Con respecto a la presencia de rentas ilegales, todos los municipios, a excepción de Valencia, tienen presencia tanto de cultivos ilícitos, como de minería ilegal. De hecho, en el reciente informe de la UNODC (2018) se alertó sobre el aumento desmesurado del área sembrada de coca en el departamento de Córdoba; el aumento entre el 2016 y el 2017 fue de 2.112 has, lo que representa un $79 \%$ más de coca sembrada. Lo

Tabla 7.

Conflictividad en la subregión Sur de Córdoba, 2018.

\begin{tabular}{|c|c|c|c|c|c|c|c|c|}
\hline Subregión & Departamento & Municipio & $\begin{array}{l}\text { Presencia de } \\
\text { cultivos ilícitos }\end{array}$ & $\begin{array}{l}\text { Presencia de } \\
\text { minería ilegal }\end{array}$ & $\begin{array}{c}\text { Presencia } \\
\text { de GAO }\end{array}$ & $\begin{array}{c}\text { Presencia } \\
\text { del ELN }\end{array}$ & $\begin{array}{l}\text { Acciones } \\
\text { armadas } \\
(2018)\end{array}$ & $\begin{array}{l}\text { Infracciones al Derecho } \\
\text { Internacional Humanitario } \\
\text { (2018) }\end{array}$ \\
\hline \multirow{6}{*}{ Sur de Córdoba } & \multirow{6}{*}{ Córdoba } & Montelíbano & Sí & Sí & Sí & No & 3 & 1 \\
\hline & & Puerto Libertador & Sí & Sí & Sí & No & 4 & 1 \\
\hline & & & & & & & & \\
\hline & & San José de Uré & Sí & Sí & Sí & No & 3 & 9 \\
\hline & & Tierralta & Sí & Sí & Sí & No & 4 & 2 \\
\hline & & Valencia & Sí & No & Sí & No & 0 & 0 \\
\hline
\end{tabular}

Fuente: Elaboración propia a partir de los datos del Centro de Pensamiento UNCaribe (2018). 
anterior resulta crítico si se tiene en cuenta que para el 2013 se había logrado llevar el número de has sembradas a 439, esto significa que entre el 2013 y el 2017 hubo un aumento porcentual de $989 \%$ de has de coca sembradas en Córdoba. Así, este departamento fue en el 2017 el segundo departamento de Colombia con más aumento porcentual en sus cultivos de coca entre los años 2016 y 2017, y el décimo en términos de cantidad de has con cultivos ilícitos ${ }^{10}$.

En lo que respecta a la presencia de actores armados, esta subregión registra GAO en todos sus municipios y en ninguno hay presencia del ELN. Lo anterior no debería sorprender teniendo en cuenta que esta guerrilla nunca tuvo arraigo importante en Córdoba y su campo de actuación estuvo más relacionado con el sur de los departamentos de Cesar y Bolívar. Empero, no debería considerarse que la inexistencia de guerrillas signifique un aliciente para los niveles de conflictividad. Por el contrario, en esta subregión se han presentado 14 acciones armadas (lo que representa cerca del 16\% del total de acciones armadas en el Caribe hasta septiembre del 2018) y 13 infracciones al DIH (lo cual es equivalente al $48 \%$ de infracciones al DIH hasta septiembre del 2018). En la figura 2 puede observarse con mayor detalle cómo esta subregión representa cerca de la mitad de las infracciones al DIH en todo el Caribe, sobrepasando con creces a todas las demás subregiones priorizadas para los PDET. De hecho, solamente en San José de Uré se ha presentado cerca del $40 \%$ de infracciones al DIH hasta septiembre del 2018.

\footnotetext{
${ }^{10}$ Es importante resaltar que el sur de Córdoba es geográficamente estratégico porque sirve como corredor de movilidad entre la Costa Caribe, el Bajo Cauca, el Norte de Antioquia y Urabá (antioqueño y chocoano), teniendo como nodo central el Parque Nacional Nudo de Paramillo. El control territorial de este parque natural resulta importante por la extensa red de conexiones terrestres y fluviales que ofrece, entre otras cosas porque conecta zonas de cultivos y centros de producción y acopio de clorhidrato de cocaína, con rutas de transporte y puntos de exportación (Trejos, 2018b).
} 

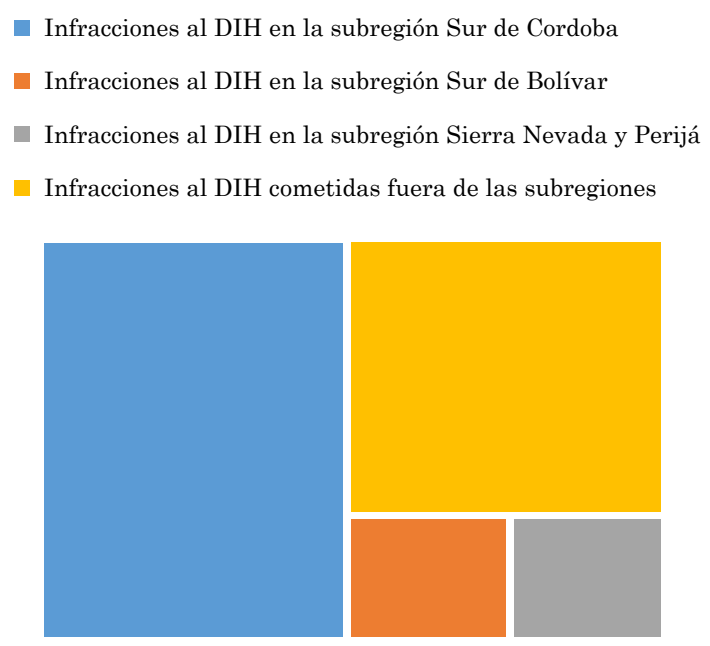

Figura 2. Infracciones al DIH por subregión: enero-septiembre, 2018. Fuente: Elaboración propia a partir del Centro de Pensamiento UNCaribe (2018).

Por otro lado, cerca del $53 \%$ de los líderes sociales asesinados en el Caribe colombiano entre enero y septiembre del 2018 ejercían sus liderazgos en alguno de los municipios de esta subregión y varios de ellos tenían alguna relación con el programa de Sustitución Voluntaria de coca. Todos los desplazamientos masivos presentados en el Caribe durante el 2018 han sucedido en San José de Uré a causa de intercambios violentos entre organizaciones armadas, y uno de los dos secuestros presentados durante este año en la región Caribe también tuvo como escenario este municipio.

La causa de esos niveles de conflictividad radica en la presencia del Clan del Golfo y una disidencia de esa organización criminal, Los Caparrapos, quienes han sido sindicados de ser los responsables de un importante número de las infracciones al DIH presentadas en la subregión. Estas organizaciones se están enfrentando en una lucha por el control de los territorios y el escenario del conflicto ha sido principalmente San José de Uré debido a su importancia estratégica para el cultivo y la posterior exportación de coca (Ardila, 2018). 
Heterogeneidad en la planeación del territorio: ¿subregiones homogéneas?

Finalmente, vale la pena mencionar de manera somera un reto para la construcción y posterior implementación de los PDET que se aparta de las concepciones violentas: la presencia de diversas visiones de desarrollo territorial entre los participantes del proceso, lo que podría complicar el éxito de los programas. Para esto, se observarán el número de Consejos Comunitarios y Resguardos Indígenas presentes en cada subregión.

En primer lugar, en las cuatro subregiones se registra la presencia de 17 Consejos Comunitarios y 22 Resguardos Indígenas, para un total de 80.902 habitantes en todos ellos. La subregión Sierra Nevada - Perijá es la que más número de ambos tiene en sus territorios: 9 Consejos Comunitarios y 19 Resguardos Indígenas. La siguen muy de lejos las subregiones Montes de María (4 Consejos Comunitarios y 1 Resguardo indígena) y la subregión Sur de Córdoba (3 Consejos Comunitarios y 2 Resguardos Indígenas). Finalmente, el Sur de Bolívar cuenta con 1 Consejo Comunitario y ningún Resguardo Indígena.

Tabla 8

Presencia de Consejos Comunitarios y

Resguardos indígenas en las subregiones priorizadas
Este panorama permite observar que, tal como se había esbozado anteriormente, la subregión Sierra Nevada - Perijá, a pesar de tener un buen panorama con respecto a las variables medidas frente a la violencia armada, se enfrenta a una amplia diversidad de actores a la hora de planear el territorio a largo plazo. Así las cosas, esto podría retrasar el proceso de discusión y adopción de un PDET, y posiblemente, el aceptado se enfrentará también ante el desacuerdo de algunos sectores. En ese sentido, los aspectos logísticos podrían entrometerse en el camino y retrasar los procesos de decisión.

\section{Conclusiones}

De las cuatro subregiones priorizadas en el Caribe colombiano para la implementación de PDET, se puede concluir que dos (Sur de Córdoba y Sur de Bolívar), presentan dinámicas importantes de conflictividad armada a la luz de las tres variables analizadas (presencia de actores armados, presencia de rentas ilegales y el grado de afectación derivado del conflicto). Con respecto a la presencia de actores armados, en el Sur de Córdoba actúan el Clan del Golfo, Los Caparrapos (un grupo armado organizado disidente del primero) y desde mediados del 2018

Número de Consejos Comunitarios y resguardos indígenas en las subregiones priorizadas

\begin{tabular}{|c|c|c|c|}
\hline Subregión & Número de Consejos Comunitarios & Número de Resguardos Indígenas & $\begin{array}{l}\text { Proyección de habitantes en } \\
\text { los resguardos } 2015 \text { (DANE) }\end{array}$ \\
\hline Montes de María & 4 & 1 & 7450 \\
\hline Sierra Nevada - Perijá & 9 & 19 & 69454 \\
\hline Sur de Bolívar & 1 & - & - \\
\hline Sur de Córdoba & 3 & 2 & 3998 \\
\hline Total & 17 & 22 & 80902 \\
\hline
\end{tabular}

Fuente: Trejos y Badillo (2018). 
hicieron presencia en el territorio grupos rearmados de exmiembros de la otrora guerrilla de las FARC. El hecho de que ningún actor haya podido establecerse de forma hegemónica en este territorio tiende a exacerbar los enfrentamientos armados y a poner en riesgo a la población civil que allí habita. Eso explicaría por qué el $48 \%$ de las infracciones al DIH en todo el Caribe tuvieron lugar en alguno de los municipios de esta subregión.

Con respecto a la presencia de rentas ilegales, en todos los municipios, a excepción de Valencia, se registra tanto minería ilegal como sembrado de cultivos ilícitos. En el informe de la UNODC (2018), además da cuenta del aumento exponencial que ha experimentado durante los últimos años. La salida de las FARC de este territorio no significó, entonces, el fin de las condiciones de conflictividad, sino la transformación de la violencia en una que ahora involucra a grupos armados con nuevas modalidades de actuación e interesados en llenar los vacíos de poder dejados por el grupo insurgente. El exagerado número de has sembradas de coca, además, coincide con el asesinato sistemático de líderes sociales, muchos de ellos relacionados con el Programa Nacional Integral de Sustitución de Cultivos Ilícitos-PNIS. En ese sentido, podría afirmarse que actores como el Clan del Golfo y Los Caparrapos (analizados principalmente en el presente texto) ejercen una lógica "econocentrada" de la violencia. Esto, en términos de Berdal y Serrano (2004), consiste en el hecho de que la mayoría de las acciones armadas de estas organizaciones solo son motivadas por las ganancias económicas (o los riesgos de perderlas).

Los líderes sociales, entonces, estarían siendo asesinados por los riesgos que significan para la producción de rentas ilícitas, debido a ser estos los encargados de conectar a la población de estos territorios con la institucionalidad estatal. Asimismo, los líderes sociales suelen servir como comunicadores de los sucesos en el territorio a través de las redes organizacionales a las que pertenecen. Los Grupos Armados Organizados (GAO) los observan como amenazas debido a que la llegada de mayor institucionalidad o la amplia comunicación del statu quo de estas 
subregiones tiende a amenazar su predominio y, por ende, las ganancias que obtienen de la explotación de los recursos y la transnacionalización de sus actividades. No sería, entonces, una cuestión predominantemente relacionada con la ideología de quienes son asesinados, sino, con las consecuencias de sus actos sobre las acciones ilícitas de los perpetradores de las mismas acciones.

Finalmente, con respecto al grado de afectación derivado del conflicto armado, el sur de Córdoba es la región en la que mayor número de acciones armadas (13\%) y de infracciones al DIH (48\%) sucedieron entre enero y septiembre del 2018 entre las subregiones comparadas. Eso indica como no solamente los actores armados están en el territorio, sino que actúan con frecuencia. Además, el abultado número de infracciones al DIH indica también que la población civil es altamente afectada por ello. No es casualidad que en el sur de Córdoba se concentren casi la mitad de las infracciones que sucedieron en todo el Caribe colombiano.

Por su parte, en el sur de Bolívar actúa también el Clan del Golfo y hay presencia de la guerrilla del ELN. En todos los municipios hacen presencia ambos o alguno de los dos actores armados. Además, la Defensoría del Pueblo alertó a mediados del 2018 de la llegada de algunos grupos disidentes de la exguerrilla de las FARC (Betín, 2018). A pesar de esto, podría pensarse, como la distancia ideológica entre las organizaciones ilegales presentes en el territorio es mucho más amplia, y por ende, este podría considerarse un espacio más propicio para conflagraciones armadas, tanto las acciones armadas como las infracciones al DIH presentadas aquí son considerablemente menores en número a las presentadas en el sur de Córdoba. Lo anterior podría deberse a esa misma lógica "econocentrada" de la violencia, y justificaría la idea de como, más allá de las motivaciones ideológicas de los actores, se asiste a un escenario en el que las ganancias económicas resultan mucho más importantes que las posiciones de los grupos armados. 
Lo anterior, sin embargo, no debería hacer pensar que se asiste a un escenario pacífico. De hecho, como se mencionó antes, cerca del $12 \%$ de las acciones armadas presentadas en el Caribe colombiano tuvieron lugar en el sur de Bolívar. Al igual que el departamento de Córdoba, Bolívar es importante para los grupos armados debido a los registros tanto de minería ilegal como de cultivos ilícitos. Asimismo, estos recursos pueden ser transportados hacia la zona costera del departamento, desde donde son exportados hacia los países de destino.

En ese sentido, puede observarse que, según la UNODC (2018), el aumento de has sembradas de coca en Bolívar durante el año 2017 fue cercano al 51\%, lo que indica que, en su territorio, se encuentra el $4 \%$ del total de cultivos presentes en todo el país. Una gran parte de esos cultivos, como puede observarse en el informe citado (UNODC, 2018), se encuentra en el sur del departamento, lo que explica que la conflictividad se concentre también allí.

Con respecto a las dos subregiones restantes, la Sierra Nevada-Perijá y los Montes de María, se puede afirmar como, aunque siguen presentándose acciones armadas e infracciones al DIH de forma esporádica, esto no responde necesariamente a la amenaza constante de algún actor armado. Lo anterior puede evidenciarse en el hecho de como en la primera región, solo dos municipios han registrado presencia del ELN (en la zona rural de Santa Marta, Magdalena y Dibulla, La Guajira) y esta no es sostenida, sino que responde a espacios de movilidad para la guerrilla (Urieles, 2018). En el caso de Montes de María, solo San Onofre ha sido alertada por la presencia del Clan del Golfo; no obstante, existe el riesgo de que la presión ejercida por la fuerza pública en medio de la operación Agamenón $\mathrm{II}^{11}$ en el

\footnotetext{
${ }^{11}$ Agamenón es la campaña militar y policial más grande de Colombia y tiene por objetivo el desmantelamiento de la estructura criminal del Clan del Golfo. Fue iniciada oficialmente el 2 de febrero de 2015 y el 1 de junio del 2017 fue renovada bajo el nombre Agamenón 2. En la segunda fase de la operación, la Armada Nacional, la Fuerza Aérea Colombiana y el Ejército se unieron a la Policía en el objetivo de desestructurar la organización criminal. Si bien la operación ha conllevado a la incautación de más de 203 toneladas de droga, 86 bajas para la organización, cerca de 600 armas incautadas y más de 1800 capturas a miembros del colectivo criminal, todavía no se puede considerar que ha cumplido con su objetivo principal: acabar con el temido grupo armado (Semana, 2018).
} 
departamento de Córdoba lleve a las organizaciones armadas a trasladarse hacia Sucre, lo cual amenazaría la seguridad de toda esta subregión.

De todos modos, a partir de las variables analizadas, puede afirmarse que los retos que pueden surgir de cara a los PDET no provienen tanto de situaciones de violencia, como de las diferencias existentes entre los actores que tendrán que coincidir en su visión sobre el territorio. Ese reto consensual se encuentra en más presente en la subregión Sierra Nevada - Perijá que en los Montes de María. Eso puede explicarse en el hecho de como mientras la segunda es una subregión en la que, al menos durante una década, las organizaciones sociales se han organizado para reconstruir el tejido social y construir paz "desde abajo", la primera es una subregión construida artificialmente para la ejecución de los PDET (como se ha mencionado anteriormente, se trata geográficamente de dos subregiones distintas que observan su espacio de forma diferenciada). Asimismo, la amplia presencia de consejos comunitarios y resguardos indígenas trae consigo la discusión acerca de las visiones del territorio y las posiciones de los distintos conglomerados sociales que tendrán que acercarse para planear la forma de transitar hacia el posconflicto.

Es importante resaltar que cerca del $65 \%$ de infracciones al DIH y cerca del $32 \%$ de acciones armadas presentadas entre enero y septiembre del 2018 han sucedido en municipios priorizados para los PDET, lo que da cuenta de como el conflicto en estos territorios no está del todo olvidado. Si bien, la mayoría de esas dinámicas conflictivas se concentran en dos subregiones, no quiere decir que en las restantes no se hayan presentado situaciones violentas; 11 de los 40 municipios priorizados tienen presencia de rentas ilegales, 14 de los 40 tienen presencia de algún actor armado ilegal y 14 de los 40 municipios han presenciado acciones armadas o infracciones al DIH entre enero y septiembre del 2018. 
Los autores consideran entre las 4 subregiones priorizadas, al sur de Córdoba como la más afectada por enfrentar mayores retos para transformar las condiciones actuales de violencia; no obstante, esto no debería significar necesariamente un retraso en el proceso de adopción del PDET (de hecho, este ya pasó la fase de discusión regional y adoptó el Plan de Acción para la Transformación Regional), sino que posiblemente signifique mayores retos para la puesta en marcha de los mismos y la consecución de sus objetivos primigenios. Por su parte, el mayor reto para las subregiones Montes de María y Sierra Nevada - Perijá consiste en superar las barreras consensuales que se imponen por la cantidad de municipios, el elevado número de organizaciones participantes y la multiplicidad de visiones que pueden aparecer. Sin embargo, al menos en el presente, no se registran situaciones de violencia que permitan hablar de escenarios de alta conflictividad.

\section{Referencias}

Aguilera, M. (2016). Serranía del Perijá: Geografía, capital humano, economía y medio ambiente. Documentos de Trabajo sobre economía regional del Banco de la República. (249). 1-134. Recuperado de http://www.banrep.gov.co/docum/Lectura_finanzas/pdf/dtser_249.pdf

Aguilera, M. (2013). Las FARC: Auge y quiebre de su modelo de guerra. Análisis Político, 26(77). 85-111.

Ardila, L. (julio 29, 2018). Los líderes (y todo el mundo) están bajo fuego en Uré. La Silla Vacía. [Silla Caribe]. Recuperado de http://lasillavacia.com/silla-caribe/los-lideres-y-todoel-mundo-estan-bajo-fuego-en-ure-67240

Arias, A. (2010). Contexto de violencia y conflicto armado. En, Misión de Observación Electoral-MOE. Monografía Político Electoral del Departamento de Cesar 1997-2007. [Online]. Recuperado de https://moe.org.co/home/doc/moe_mre/CD/ PDF/cesar.pdf 
Ávila, A. (septiembre 12, 2018). La mafia que maneja la Sierra Nevada: Los Pachencas y la Oficina Caribe. Semana. [Opinión]. Recuperado de https://www.semana.com/opinion/articulo/la-mafia-que-maneja-la-sierra-nevada-por-ariel-avila/582788

Berdal, M. y Serrano, M. (2004). Introducción. En M. Berdal, y M. Serrano (coord.), Crimen transnacional organizado y seguridad internacional: cambio y continuidad (13-26). México, D.F.: Fondo de Cultura Económica-FCE.

Betín, T. (agosto 30, 2018). Defensoría advierte presencia de grupos ilegales en el sur de Bolívar. El Heraldo. [Bolívar]. Disponible en: https://www.elheraldo.co/bolivar/defensoria-advierte-presencia-de-grupos-ilegales-en-el-sur-de-bolivar-536294

Centro de Pensamiento UNCaribe. (2018). Observatorio de Dinámicas del Conflicto Armado en la Región Caribe. [Base de datos]. Disponible en https://www.uninorte.edu.co/web/ uncaribe/observatorio

Daniels, A. (2012). Las políticas públicas para las regiones en transición en el caribe colombiano. En R. Román, A. Vidal y J. Caro. (Comps.), Imperios, mercados y multiculturalidad en el Caribe. Memorias del II Congreso Internacional de Estudios Caribeños. (187-204). San Andrés: Universidad Nacional de Colombia, Universidad del Norte y Universidad del Magdalena.

Daniels, A., Menco, D., Jiménez, J. y Peralta, M. (2011). El laboratorio de Paz en Los montes de María: Una aproximación a su contexto. En A. Daniels y A. Múnera, Los Montes de María: Región, conflicto armado y desarrollo productivo. (572). Cartagena: Pluma de Mompox.

Defensoría del Pueblo (2018b). Alerta Temprana $N^{\circ}$ 045-18. Recuperado de https://opinioncaribe.com/wp-content/uploads/2019/01/AT-Nº-045-18-MAG-Cienaga2c-Santa-Marta-y-LAG-Dibulla.pdf 
El Heraldo.com (2018). San Onofre está en alto riesgo por presencia de AGC. El Heraldo. [Redacción Regionales]. Recuperado de https://www.elheraldo.co/sucre/san-onofre-estaen-alto-riesgo-por-presencia-de-agc-525310

Esser, F. y Vliegenthart, R. (2017). Comparative Research Methods. En J. Matthes (Ed.) The International Encyclopedia of Communication Research Methods, (1-22). New Jersey: John Wiley \& Sons.

García, P., Irreño, Y. y Gutiérrez, A. (2018). La renovación del territorio: los Planes de Desarrollo con enfoque territorial y la planeación local. Barranquilla: UNCaribe, UniNorte.

Gutiérrez, A. (2018). Sur de Bolivar: Contexto geográfico, socioeconómico e institucional. Barranquilla: Centro de Pensamiento UNCaribe, Universidad del Norte.

Iguaran, A. (mayo 7, 2017). La guerra de 'los Pachenca' y el 'Clan del Golfo' para 'tomarse' a Barranquilla. El Heraldo. [Judicial]. Recuperado de https://www.elheraldo.co/judicial/ la-guerra-de-los-pachenca-y-el-clan-del-golfo-para-tomarsebarranquilla-357839

Irreño, Y. y Martínez, F. (2018). Dinámicas del Conflicto Armado en el Sur de Córdoba. Barranquilla: Centro de Pensamiento UNCaribe, Universidad del Norte.

Landman, T. (2000). Issues and methods in comparative politics. Milton Park: Routledge.

Marsh, D. y Stoker, G. (1997). Teoría y Métodos de la Ciencia Política. Madrid: Alianza.

MOE. (2018). Programas de Desarrollo con Enfoque Territorial. [Primer informe de observación]. Bogotá, D.C.: MOE. Recuperado de https://moe.org.co/wp-content/uploads/2018/07/ PDET_Final_Web-3.pdf

Observatorio de Paz y Conflicto-OPC. (2012). Estructuras de Autodefensas y Proceso de Paz en Colombia. Bogotá, D.C.: Universidad Nacional de Colombia, Embajada de Suecia. Recuperado de http://www.humanas.unal.edu. 
co/observapazyconflicto/files/4814/3594/1011/informeestructurasdeautodefensasyprocesode__pazen_colombia_ version15_02_12_final.pdf

Observatorio del Caribe Colombiano (2013). Caracterización del Caribe colombiano. Disponible en http://www.ocaribe.org/ region-caribe?la=es

ONU. UNODC. (2018). Colombia: Monitoreo de territorios afectados por cultivos ilicitos 2017. Resumen ejecutivo.

ONU. PNUD. (2011). Regiones en conflicto: comprender para transformar. Bajo Cauca, Huila, Meta, Montes de María y Nariño. Informe de Desarrollo Humano Colombia 2011. Disponible en: http://www.co.undp.org/content/colombia/es/ home/library/human_development/regiones-en-conflicto--comprender-para-transformar-.html

ONU. PNUD. (2010). Montes de María: Análisis de la conflictividad. Disponible en https://info.undp.org/docs/pdc/Documents/COL/00058220_Analisis\%20conflcitividad\%20 Montes\%20de\%20Maria\%20PDF.pdf

República de Colombia. Ministerio de Agricultura y Desarrollo Rural. (28 de mayo de 2017). Por el cual se crean los Programas de Desarrollo con Enfoque Territorial (PDET). [Decreto 893]. DO: 50247.

República de Colombia. Oficina del Alto comisionado para la paz. (s.f.). El plan de desarrollo de tu municipio, ¿construye paz? ¿ya lo viste?. Recuperado de http://www.altocomisionadoparalapaz.gov.co/Documents/informes-especiales/plandesarrollo-territorial/index.html

República de Colombia. Jurisdicción Especial para la Paz. (24 de noviembre de 2016). Acuerdo final para la terminación del conflicto y la construcción de una paz estable y duradera. [Acuerdo Final]. Recuperado de https://www.jep.gov. co/Paginas/Normativa/Acuerdo-Final.aspx

República de Colombia. Vicepresidencia de la República. Observatorio de Derechos Humanos y Derecho Internacional 
Humanitario. (2009). Dinámica de la violencia en el departamento de Córdoba 1967-2008. Recuperado de https://bit. ly/2qwH0x7

República de Colombia. Vicepresidencia de la República. Observatorio de Derechos Humanos y Derecho Internacional Humanitario. (2008). Panorama actual de la región de Montes de María y su entorno. Recuperado de http://historico. derechoshumanos.gov.co/Observatorio/Publicaciones/documents/2010/Estu_Regionales/04_03_regiones/montes/montes.pdf

Semana. (26 de febrero de 2018). Las cifras de Agamenón, la operación más grande contra el crimen colombiano. Revista Semana. [Infografía]. Recuperado de https://www.semana.com/nacion/articulo/balance-de-la-operacion-agamenoncontra-el-clan-del-golfo/558357

Trejos, L. (2018a). Riesgo electoral asociado a la presencia de actores armados y rentas ilegales en el Caribe colombiano: "Entre la herencia paramilitar y la marginalidad insurgente”. Barranquilla: Centro de Pensamiento UNCaribe.

Trejos, L. (2018b). La guerra persiste en los sures del caribe. La Silla Vacia. [La silla caribe]. Disponible en: https:// lasillavacia.com/silla-llena/red-caribe/historia/la-guerrapersiste-en-los-sures-del-caribe- 65329

Trejos, L. y Badillo, R. (2018). Circunscripciones Transitorias Especiales de Paz: La materialización de la paz territorial. Barranquilla: Centro de Pensamiento UNCaribe, Universidad del Norte.

Trejos, L. (2017). Posacuerdo en el caribe. Ilegalidad y nuevas violencias. Revista Palimsesto 9(12). 76-96.

Trejos, L. (2016). Hipótesis explicativas de la derrota estratégica de la insurgencia armada en el Caribe colombiano: los casos de las FARC-EP y el ELN. Revista de Derecho Uninorte, (45). 346-377. https://doi.org/10.14482/dere.45.7982 
Urieles, R. (mayo 23, 2018). Refuerzan vigilancia en corregimiento de Minca por presencia del ELN. El Tiempo. [Santa Marta]. Recuperado de https://www.eltiempo.com/colombia/ otras-ciudades/mas-seguridad-en-minca-por-presencia-deleln-221156

Velásquez, F. y González, E. (2003). ¿Qué ha pasado con la participación ciudadana en Colombia?. Bogotá, D.C.: Fundación Corona.

VerdadAbierta.com (febrero 12, 2014). Hernán Giraldo: máquina de guerra en la Sierra Nevada de Santa Marta. Verdad Abierta. [Justicia y Paz]. Recuperado de https://verdadabierta.com/hernan-giraldo-maquina-de-guerra-en-la-sierra-nevada-de-santa-marta/

VerdadAbierta.com (septiembre 2, 2010). ¿Cómo se fraguó la tragedia de los Montes de María? Verdad Abierta. [La Historia]. Recuperado de https://verdadabierta.com/icomo-sefraguo-la-tragedia-de-los-montes-de-maria/ 
Luis Trejos Rosero es Doctor en Estudios Americanos con mención en Estudios Internacionales (IDEA/USACH). Profesor investigador del Departamento de Ciencia Política y Relaciones Internacionales de la Universidad del Norte (Barranquilla, Colombia). Miembro del grupo de investigación "Agenda Internacional" de la Universidad del Norte y Director del Centro de Pensamiento UNCaribe (Barranquilla, Colombia). https://orcid. org/0000-0003-4988-8576

Reynell Badillo Sarmiento es Internacionalista con énfasis en Ciencia Política y Relaciones Internacionales de la Universidad del Norte (Barranquilla, Colombia). Investigador del Centro de Pensamiento UNCaribe (Sabanagrande, Colombia). https://orcid.org/0000-0002-3576-8533

Yiseth Irreño Quijano es Politóloga con énfasis en Políticas Públicas y Gobierno de la Universidad del Norte (Barranquilla, Colombia). Investigadora del Centro de Pensamiento UNCaribe. (Barranquilla, Colombia). https://orcid.org/0000-0001-96206465 